\title{
AVALIAÇÃO FISICA EM MENINAS PARTICIPANTES DO PROJETO SEGUNDO TEMPO DA CIDADE DE AMERICANA-SP
}

\author{
Samuel Bento da Silva \\ Jose Francisco Daniel \\ Rodrigo Siqueira \\ Rafael Correa \\ Lucila Santos da Silva
}

\section{Resumo}

O objetivo do estudo foi verificar se há correlação entre medidas antropométricas e capacidades físicas de 59 meninas participantes do Projeto Segundo Tempo da cidade de Americana-SP, com idades entre 10 a 19 anos. A correlação entre os resultados das medidas antropométricas e os resultados de testes físicos, foi verificado através do coeficiente de Spearman. Os resultados indicam que as medidas antropométricas influenciam as capacidades físicas observadas. Também foram verificadas correlações, estatisticamente significantes entre os resultados dos testes das capacidades físicas. Todos os testes com exceção a flexibilidade se correlacionaram entre si.

\section{Palavras-Chave}

Capacidades físicas; Medidas antropométricas; Projeto Segundo Tempo.

\section{MENINAS PARTICIPANTS IN PHYSICAL EVALUATION PROJECT SECOND TIME OF THE CITY OF AMERICAN-SP}

\begin{abstract}
The mains goal of this study is to verify if there is a correlation between measures anthropometric measures and capacities physics of 59 girls participants of the screen program Segundo Tempo from city of Americana, with ages among 10 the 19 years. The correlation among the results from the measures anthropometric and results of tests was checker via the coefficient of Spearman. The result indicates as the measures anthropometric influence the capabilities physics observer. Also have been checker correlations significant among the results from the tests from the capacities physics. All of the tests with exception the flexibility if correlate among oneself.
\end{abstract}

\section{Key-Words}

Physical capacities; Anthropometric measures; Program second stage . 


\section{INTRODUÇÃO}

$\mathrm{O}$ momento atual limita as possibilidades de vivencia motora infantil, devido a estrutura organizacional das cidades, altos índices de violência, pouco tempo destinado ao convívio familiar, em especial, nas atividades físicas. As aulas de Educação Física são realizadas em apenas duas horas aulas semanais, muito pouco para as reais necessidades.

Alguns projetos públicos são desenvolvidos em diversas cidades, no sentido de proporcionar acesso democrático as praticas esportivas, deteç̧ão de talentos esportivos, entre outros. O Programa Segundo Tempo, do Ministério do Esporte, através de parcerias, estimula o desenvolvimento do esporte educacional no contra-turno escolar. Tem como finalidade o desenvolvimento de valores sociais, a melhoria das capacidades físicas e habilidades motoras, a melhoria da qualidade de vida, diminuição da exposição aos riscos sociais e a conscientização da pratica esportiva, assegurando o exercício da cidadania (SEGUNDO TEMPO, 2007).

Independentemente dos objetivos de qualquer programa físico esportivo, deve se pautar pela qualidade em todas suas possibilidades. Neste sentido, uma das possibilidades e o acompanhamento do estado morfológico e motor do publico envolvido, tanto para a deteç̧ão de problemas de obesidade e desnutrição, e baixo nível das capacidades físicas, procurando soluciona-los, como para a verificação da evolução do trabalho.

Com relação ao aspecto morfológico, índices simples como a medida da massa, estatura e envergadura, proporcionam importantes informações do estado atual do individuo, e se correlacionadas as capacidades físicas, ainda mais importantes.

Neste sentido, as atividades em que a massa muscular deve ser movida no espaço, principalmente quando envolve as capacidades físicas velocidade, resistência, equilíbrio, agilidade e os saltos, são afetadas negativamente pelo nível elevado da massa, em especial de gordura (WILMORE; COSTILL, 2001).

No inicio da puberdade não há grande desenvolvimento da massa muscular, podendo ser feita correlação entre a simples medida da massa e o desempenho em determinadas capacidades físicas. Em trabalho que foi verificada a porcentagem de gordura, ao correlacioná-la as variáveis do desempenho motor em crianças e adolescentes de Londrina - PR, Guedes e Guedes (1996) verificaram que quanta maior a quantidade de gordura corporal apresentada, menor o desempenho motor. 
Com relação ao desempenho motor, discorreremos, brevemente sobre as capacidades físicas velocidade, agilidade, forca, flexibilidade e resistência aeróbia.

A velocidade e uma capacidade neuromuscular presente em todas as situações, nos vários esportes, sendo que tudo se orienta por ela (BARBANTI, 1997) tornando-se seu principal fator de rendimento esportivo (CUNHA, 2000). Nos esportes coletivos e uma capacidade múltipla, que depende da rápida reação, do manuseio da situação, da rapidez em iniciar o movimento e dar seqüência ao mesmo, da aptidão com a bola, do rápido reconhecimento e utilização das respectivas situações (WEINECK, 1999). Mas, devido as curtas distancias e trocas constantes de direção realizadas nestes, dificilmente consegue-se desenvolver picos de velocidade, com maior ênfase na capacidade de aceleração.

$\mathrm{Na}$ fase de formação dos atletas, os progressos observados com a velocidade se devem tanto ao aumento da eficiência e controle do movimento e dos gestos desportivos, como ao aumento da forca. Ao final desta fase os ganhos acentuados desta capacidade se devem, sobretudo, ao aumento da capacidade anaeróbia e da forca de explosão (FRISSELLI; MANTOVANI, 1999).

A agilidade e definida "como uma variável neuromotora caracterizada pela capacidade de realizar trocas rápidas de direção, sentido e deslocamento da altura do centra de gravidade de todo o corpo ou parte dele" (MATSUDO, 1984). E uma capacidade física importantíssima em diversos esportes, como handebol, basquetebol, voleibol, etc., assim como em diversas situações da vida diária. E interdependente da forca, velocidade, flexibilidade e coordenação (BOMPA, 2002). Foi verificado por Seabra, Maia e Garganta (2001), em estudo realizado com futebolistas, que o treinamento exerce influencia positiva sobre esta capacidade.

A forca esta sempre combinada com outros fatores determinantes do desempenho (WEINECK, 1999). sendo definida "como a capacidade de superar a resistência externa, as custas dos esforços musculares". E considerada "como a condição para assegurar a velocidade dos movimentos (deslocamentos) do esportista", influenciando, consideravelmente a velocidade, resistência e agilidade (VERKOSHANSKY, 2001).

O aumento da forca acompanha os processos de maturação e crescimento (aumento do tamanho das estruturas ósseas e aumento da massa muscular) (SILVA; ALVES, 1998), sendo a adolescência a fase apropriada para o seu desenvolvimento e da potencia muscular em paralelo a coordenação. Nesta fase 
ocorrem os maiores aumentos da forca muscular, alem do reforço ligamentar e ósseo propicio para o trabalho de desenvolvimento da forca geral, da forca rápida e da potencia muscular (BARATA, 1999). Jovens esportistas na faixa etária dos 12 aos 16 anos de idade, apresentam diferenças significativas no nível de forca em relação aos demais adolescentes da mesma idade, devido ao efeito positivo do treinamento (SEABRA; MAIA; GARGANTA, 2001), que aumenta a ativação neuromuscular (SILVA; ALVES, 1998).

A flexibilidade e definida como a capacidade física "expressa pela maior amplitude possível do movimento voluntário de uma articulação, ou combinações de articulações num determinado sentido". O grau de flexibilidade e determinado pela amplitude articular e pela elasticidade ou extensibilidade muscular (DANTAS, 1985). E de grande importância para as modalidades de forca; e também associado a prevenção de lesões (GOMES; MONTEIRO; VIANNA, 1997) e a velocidade devido a necessidade de se possuir amplitude adequada de movimentos nos ombros, quadris e tornozelos (DINTIMAN, WARD e TELLEZ, 1999).

$\mathrm{O}$ desenvolvimento da flexibilidade tem efeitos positivos sobre fatores físicos do desempenho esportivo e sobre a técnica esportiva (WEINECK, 1999). Neste sentido, a flexibilidade e importante como base de apoio também para a performance motora geral e, e a única capacidade física que tem sua fase sensível adequada no momento da passagem da infância para a adolescência, para decrescer logo em seguida (FRISSELLI; MANTOVANI, 1999).

Os ritmos mais altos de acréscimo da flexibilidade situam-se entre os 9 e os 14 anos e considera-se a idade entre os 15 e os 17 anos a mais tardia para o desenvolvimento desta capacidade. Os índices superiores de flexibilidade registram-se entre os 12 e os 17 anos de idade (ZAKHAROV, 1992).

O sistema aeróbio e fisiologicamente associado ao $\mathrm{VO}_{2} \max$ e definido como a capacidade do organismo suprir aerobicamente o trabalho muscular, associado as condições de os tecidos utilizarem o oxigênio na sustentação dos esforços físicos (SHARKEY, 1997). Este sistema trata da integração entre o sistema cardiovascular, respiratório e muscular, e caracteriza a capacidade de todo o organismo responder ao exercício (BARBANTI, 2001).

$\mathrm{O} \mathrm{VO}_{2}$ max representa a mais alta captação de oxigênio alcançada por um individuo respirando ar atmosférico ao nível do mar (POWERS; HOWLEY, 2000), sendo esta variável e utilizada para a 
determinação da capacidade aeróbia relacionada a saúde e a performance (FOSS; KETEYIAN, 2000).

A capacidade aeróbia depende de fatores centrais e fatores periféricos. Os fatores centrais são relacionados a capacidade combinada dos mecanismos pulmonar, cardíaco, vascular, sanguíneo e celular para transporte de oxigênio ate o mecanismo aeróbio dos músculos. Os fatores periféricos dependem da potencialidade do individuo em liberar energia, por intermédio dos processos oxidativos, na realização de trabalho muscular por período de tempo relativamente longo, ou seja, da capacidade química dos tecidos musculares para a utilização do oxigênio (GUEDES, 2002).

Apos os 12 anos de idade há um aumento da sensibilidade ao treino do sistema cárdio-circulatorio, com possibilidade de aumento da capacidade aeróbia (BORMS, 1986).

Conforme apontado na revisão, os estudos observando capacidades físicas em crianças e adolescentes tem sido freqüentes no Brasil e em outros paises, muitos são os pesquisadores preocupados com essas questões. O presente estudo teve como objetivo avaliar as medidas antropometricas e as capacidades físicas de crianças e adolescentes que participam do Projeto Segundo Tempo que tem como objetivo a formação e inclusão social e correlacionar os resultados desses testes observando suas relações. Essa analise foi baseada em um outro objetivo do projeto que e apontar crianças que possuem potencial esportivo e cadastra-las em um banco de dados criado pelo Proesp -BR.

\section{METODOLOGIA}

Foram avaliadas 59 meninas participantes do projeto segundo tempo da modalidade handebol do núcleo centra cívico da cidade de Americana-SP, com idades entre 10 a 19 anos.

As meninas freqüentam o projeto três dias por semana (segunda, quarta e sexta feira) com aulas de 01h30mim. Os testes foram feitos em dois dias, o processo de avaliação foi realizado com duracao de 02h00min horas aproximadamente de testes. As aplicações dos testes foram feitas de acordo com a Cartilha do Ministério do Esporte elaborada pelo PROESP (Projeto Esporte Brasil) desenvolvido pelo Setor de Pedagogia do Esporte do CENESP-UFRGS.

Em relação as medidas antropometricas, foi verificada a estatura, a massa corporal e a envergadura dessas crianças, para isso foram usadas fitas métricas da marca Butter Fly Brand afixadas a parede, uma balança analógica da marca Lupa, e uma trena de fibra de vidro fechada de $20 \mathrm{~m}$ Brasfort. 
As capacidades físicas foram verificadas através dos testes de flexibilidade -sentar e alcançar, abdominal, salto horizontal, arremesso de medicineball $(2 \mathrm{~kg})$, agilidade - quadrado, velocidade - $20 \mathrm{~m}$ e resistência - 9 minutos.

A correlação entre os resultados das medidas antropometricas e os resultados de testes físicos, foi verificado através do coeficiente de Spearman, utilizando-se o pacote estatístico SPSS for Windows 14.0.

\section{RESULTADOS E DISCUSSÃO}

Os valores médios encontrados nos resultados dos testes foram: Estatura 1,64m; Massa corporal $53 \mathrm{~kg}$.; Envergadura 1,63m; Flexibilidade $46 \mathrm{~cm}$; Abdominais 29 repetições; Salto Horizontal 1,32m; Arremesso de medicinebol 2,80m; Teste de agilidade (Quadrado) 7,60seg; Velocidade (20metros) 3,94seg; Resistência aeróbia (9 minutos) 1340m.

Houve correlação estatisticamente significante, considerando-se o coeficiente de spearman, para $p<0,01$, entre estatura e os resultados dos testes de abdominais, salto horizontal, arremesso de medicineball, agilidade, velocidade e resistência. Os achados indicam que quanta maior a estatura das meninas, melhores foram os resultados nos testes citados. Apenas para a flexibilidade que a correlação não foi significante. Um dos fatores que podem ter influenciado nesse resultado é o fato de que nesse período o crescimento ósseo e maior que o crescimento muscular interferindo negativamente na flexibilidade, e no caso da resistência o fato da menina ser mais alta faz com que o resultado seja inferior.

Também houve correlação, estatisticamente significante, considerando-se o coeficiente de spearman, para $\mathrm{p}<0,01$, entre massa corpórea e os resultados dos testes de abdominais, salto horizontal, arremesso de medicineball, agilidade e velocidade. Assim como a estatura, a massa corpórea influenciou positivamente na maioria dos testes. Apenas na flexibilidade e na resistência aeróbia a massa corpórea foi um fator negativo. Uma das causas desse resultado pode ser que com o nível de massa muscular aumentado, consequentemente o nível de forca também aumenta, melhorando os resultados nos testes de forca e prejudicando os de resistência.

A envergadura apresentou correlação estatisticamente significante, considerando-se o coeficiente de spearman, para $\mathrm{p}<0,01$ entre os resultados dos testes de flexibilidade, abdominais, salto horizontal, arremesso de medicineball, agilidade, velocidade e resistência. Todas as correlações entre a envergadura 
e os resultados dos testes foram estatisticamente significantes, ou seja, essa variável influenciou diretamente nos testes, no caso da flexibilidade, a influencia foi negativa. Diferente do senso comum, o resultado mostra que a menina com maior envergadura foi a que apresentou um pior resultado no teste de flexibilidade, como já discutido anteriormente, o período de maturação óssea e diferente do período de maturação muscular e esse fenômeno pode trazer prejuízos a flexibilidade do avaliado.

Tabela 1 - Coeficiente de Spearman para Correlação entre os Resultados das Medidas Antropometricas e os Resultados dos Testes Físicos.

\begin{tabular}{lccccccc}
\hline & Flexibilidade & Abdominal & Salto H. & A. Medicine & Quadrado & Velocidade & Resistencia \\
\hline Estatura & -0.205 & $0.411^{*}$ & $0.564^{*}$ & $0.648^{*}$ & $-0.429^{*}$ & $-0.360^{*}$ & $-0.350^{*}$ \\
$\begin{array}{l}\text { Massa } \\
\text { Corpórea }\end{array}$ & -0.154 & $0.350^{*}$ & $0.365^{*}$ & $0.701^{*}$ & $-0.323^{*}$ & $-0.330^{*}$ & 0.214 \\
Envergadura & $-0.322^{*}$ & $0.417^{*}$ & $0.506^{*}$ & $0.643^{*}$ & $-0.386^{*}$ & $-0.370^{*}$ & $0.339^{*}$ \\
\hline
\end{tabular}

${ }^{*} \mathrm{p}<0,01$

Outros valores de correlação, estatisticamente significativa foram verificados quando os resultados das capacidades físicas foram relacionados entre si. Os resultados do teste de flexibilidade não se correlacionaram com nenhum outro resultado. Em relação ao teste de abdominais, seus resultados se correlacionaram significativamente para $\mathrm{p}>0,01$ com os resultados do salto horizontal (0.532), arremesso de medicinebol (0.578), quadrado (-0.511), velocidade (-0.453) e com a resistência (0.352).

No salto horizontal os resultados se correlacionaram significativamente para $p>0,01$ com o teste de abdominais (0.532), arremesso de medicinebol (0.635), quadrado (-0.788), velocidade (-0.602) e com a resistência (0.378).

$\mathrm{O}$ arremesso de medicinebol se correlacionou com o teste de abdominais (0.578), salto horizontal (0.635), quadrado (-0.577), velocidade (-0.465) e com a resistência (0.323).

Os resultados do teste do quadrado se correlacionaram com, o teste de abdominais (-0.511), arremesso de medicinebol (-0.577), salto horizontal (-0.788), velocidade (0.640) e com a resistência (-0.404). Observando os valores do coeficiente de correlação nota-se que as alunas que obtiveram bons resultados no teste de agilidade não repetiram o sucesso nos outros testes.

O ultimo teste, o de 9minutos, teve seus resultados, significativamente correlacionados para $\mathrm{p}>0,01$ com o teste de abdominais (0.532), arremesso de medicinebol (0.323), salto horizontal (0.378), quadrado (-0.404) e velocidade (-0.343). 
Tabela 2- Coeficiente de Spearman para Correlação entre os Resultados dos Testes Físicos.

\begin{tabular}{|c|c|c|c|c|c|c|}
\hline Abdominais & Abdominais & $\begin{array}{c}\text { A. } \\
0.578^{*}\end{array}$ & $\begin{array}{l}\text { Salto H. } \\
0.532 *\end{array}$ & $\begin{array}{c}\text { Velocidade } \\
-0.453^{*}\end{array}$ & $\begin{array}{l}\text { Quadrado } \\
-0.511^{*}\end{array}$ & $\begin{array}{c}\text { Resistência } \\
0.532^{*}\end{array}$ \\
\hline A. Medicine & $0.578^{*}$ & & $0.635^{*}$ & $-0.465^{*}$ & $-0.577^{*}$ & $0.323^{*}$ \\
\hline Salto H. & $0.532 *$ & $0.635^{*}$ & & $-0.602 *$ & $-0.788^{*}$ & $0.378^{*}$ \\
\hline Velocidade & $-0,453 *$ & $-0.465^{*}$ & $-0.602 *$ & & $0.64 *$ & $-0.343^{*}$ \\
\hline Quadrado & $-0.511 *$ & $-0.577 *$ & $-0.788^{*}$ & $0.64 *$ & & $-0.404 *$ \\
\hline Resistencia & $0.532 *$ & $0.323^{*}$ & $0.378^{*}$ & $-0.343^{*}$ & $-0.404 *$ & \\
\hline
\end{tabular}

\section{CONSIDERAÇÕES FINAIS}

Os resultados indicam que as medidas antropometricas de estatura, massa corporal e envergadura influenciam as capacidades físicas observadas. A estatura influenciou nos resultados dos testes de abdominais, salto horizontal, arremesso de medicineball, agilidade, velocidade e resistência. A massa corpórea influenciou os resultados dos testes de abdominais, salto horizontal, arremesso de medicineball, agilidade e velocidade. A envergadura apresentou correlação entre os resultados dos testes de flexibilidade, abdominais, salto horizontal, arremesso de medicineball, agilidade, velocidade e resistência. Foram verificadas correlações, estatisticamente significantes entre os resultados dos testes das capacidades físicas. Todos os testes, com exceção a flexibilidade se correlacionaram entre si.

Analisando os resultados do presente estudo, nota-se que as medidas antropometricas influenciam positiva e negativamente nas capacidades físicas das crianças e adolescentes observadas. Outros estudos são necessários para que se obtenha novas respostas sobre essas influencias. A aplicação de um questionário sobre as atividades diárias ou dados como porcentagem de gordura corporal poderiam fornecer dados ainda mais concretos para novos estudos.

\section{REFERENCIAS}

BARATA, A. O treino das capacidades condicionais em jovens desportista. Treino Desportivo. v. 2, n. 3, p. 31-34, nov. 1999.

BARBANTI, V. J. Teoria e prática do treinamento esportivo. São Paulo: Edgard Blurcher. 1997.

BOMPA, T.O. Periodização: teoria e metodologia do treinamento. São Paulo: Phorte, 2002.

CUNHA, L. O treino da velocidade no jovem. Treino Desportivo. v. 3, p. 26-35, out. 2000.

DINTIMAN, G.; WARD, B.; TELLEZ, T. Velocidade nos esportes: programa n. 1 para atletas. São Paulo: Manole, 1999.

FRISSELLI, A.; MANTOVANI, M. Futebol: teoria e prática. São Paulo: Phorte, 1999. 
GOMES, A. C.; MONTEIRO, G. A.; VIANNA, P. M. Alongamento Treinamento Desportivo, v. 2, n. 1, p. 91-94, 1997.

GUEDES, D. P.; GUEDES, J. E. R. P. Associação entre variáveis do aspecto morfológico e desempenho motor em crianças e adolescentes. Rev. Paul. Educ. Fis. v. 10, n. 2, p. 99-112, jul/dez. 1996. MATSUDO, V. K. R. Testes em ciências do esporte. São Caetano do Sul: Celafiscs. 1984.

SEABRA, A.; MAIA, J. A.; GARGANTA, R. Crescimento, maturação, aptidão física, forca explosiva e habilidades motoras especificas. Estudo em jovens futebolistas e não futebolistas do sexo masculino dos 12 aos 16 anos de idade. Rev. Port. Cien. Desp. v. 1, n. 2, p. 22-35, 2001.

SILVA, A.; ALVES, F. Treino de força em crianças e jovens. Treino desportivo. v. 1, n. 3, p. 37-42, out. 1998.

VERKOSHANSKY, Y. V. Treinamento Desportivo: teoria e metodologia. Porto Alegre: Artmed, 2001.

WEINECK, J. Treinamento ideal. São Paulo: Manole, 1999.

WILMORE, J. H.; COSTILL, D. L. Fisiologia do esporte e do exercício. São Paulo: Manole, 2001. 


\section{Samuel Bento da Silva}

E-Mail: sa_trabalhos@yahoo.com.br

Jose Francisco Daniel

Rodrigo Siqueira

Rafael Correa

Lucilay Santos da Silva

Projeto Segundo Tempo/ Federação Paulista de Xadrez/ Secretaria de Esportes de Americana-SP

Apoio Financeiro: Programa Segundo Tempo

Federação Paulista De Xadrez

Secretaria De Esportes De Americana

Área Temática: Metodologia do Treinamento Desportivo.

Avaliação de Rendimento.

\section{Referência do artigo}

\section{ABNT}

SILVA, S. B., et al. Avaliação fisica em meninas participantes do projeto segundo tempo da cidade de americana-sp. Conexões, v. 6, (edição especial), p. 1-10, 2008

\section{APA}

Silva, S. B., Daniel, J. F., Siqueira, R., Correa, R., \& Silva, L. S. (2008). Avaliação fisica em meninas participantes do projeto segundo tempo da cidade de Americana-SP. Conexões, 6 (ed. especial), 1-10.

\section{VANCOUVER}

Silva SB, Daniel JF, Siqueira R, Correa R, Silva LS. Avaliação fisica em meninas participantes do projeto segundo tempo da cidade de Americana-SP. Conexões, 2008; 6 (ed. especial): 1-10. 